\title{
Time Varying Back Propagating Algorithm for MIMO Adaptive Inverse Controller
}

\author{
Ibrahim Mustafa Mehedi \\ Center of Excellence in Intelligent Engineering Systems (CEIES) \\ Department of Electrical and Computer Engineering \\ King Abdulaziz University, Jeddah - 21589, Saudi Arabia
}

\begin{abstract}
In the field of automatic control system design, adaptive inverse is a powerful control technique. It identifies the system model and controls automatically without having prior knowledge about the dynamics of plant. In this paper neural network based adaptive inverse controller is proposed to control a MIMO system. Multi layer perception and back propagation are combinedly used in this investigation to design the NN learning algorithm. The developed structure represents the ability to identify and control the MIMO system. Mathematical derivation and simulation results for both plant identification and control are shown in this paper. Further, to prove the superiority of the proposed technique, performances are compared with recursive least square (RLS) method for the same MIMO system. RLS based adaptive inverse scheme is discussed in this paper for plant identification and control. Also the obtained simulated results are compared for both plant parameter estimation and tracking trajectory performance.
\end{abstract}

Keywords-Adaptive inverse control; neural network; MIMO; multilayer perception

\section{INTRODUCTION}

Prior knowledge is an important factor for almost every conventional control system. Such as in continuous time system, number of poles and zeros or the limit of upper bounds on the order of the plant are assumed to be known [1], [2], [3], [4]. Again the known time delay is crucial for discretetime systems [5], [6], [7]. To overcome these difficulties, the adaptive control methods were developed. Because it can work even if the system structure and critical parameters are unknown [6], [10]. There are several approaches are proposed to develop the adaptive controllers [8] and already been implemented for different robotic applications. Such an application is presented in [9]. In this example work, an adaptive neural network control approach is used to enhance the performance of a flexible manipulator. Adaptive controllers, based on selfTuning method were proposed to avoid the problem of uncancellable zeros for the system transfer function [11], but the reference model of the adaptive control depends on transfer function of the plant. Due to this problem, the desired output is not independent of the plant characteristics. The adaptive inverse control is one of the solutions to overcome these difficulties. It is a method to design an automatic control system. It learns over time to control a particular dynamic system [12]. Adaptive filtering technique proceeds with three concurrent learning steps and eventually develops adaptive inverse control method [13]. At the beginning, the modeled adaptive plant identifies the system dynamics. Next, the control dynamics of the plant is learned by a feed-forward controller. Finally, the disturbance affecting the plant is canceled by an adaptive feedback disturbance canceler. These controllers approximately compensate the effect of numerator polynomial at the output with the help of approximate inverse of the plant [4], [10]. The desired trajectory is approximately followed by the output of the plant with some delay which can be estimated.

The plant dynamics is controlled by several neural network (NN) approaches. A dual step controller based on neuralnetwork is used to obtain feedback linearizion and learning of the plant dynamics [14]. The calculation complexities of computing inverse dynamic are reduced by using neural network method. It also improves the precision by learning procedure. A different neural network technique is considered using a feed-forward inverse recurrent method based PD controller [15]. Inversion error is compensated and disturbances are rejected using this technique. Past investigations show the better performance while using neural network controllers for controlling the nonlinear plant dynamics [16]. Gain tuning is also performed for PD controller using NN [17].

All of these techniques used for neural network controllers have firm restrictions. In general, they require to know the fairly precise plant model before hand. Adaptive inverse control technique is considered in this paper, which is based on neural network using multi layer perception for MIMO system. A simplified architecture of the NN models are incorporated in which the modeling of the system approximate inverse of the plant are obtained directly. Then the approximate inverse model is used for the learning process to control the plant dynamics.

The rest of the paper is presented as follows: The problem stated for the purpose of this investigation is mentioned in the the next section. Architecture of adaptive inverse control technique is explained in Section 3 for multi input and multi output (MIMO) system. Multilayer perception based neural network concept is discussed in Section 4. In Section 5, back propagation based learning algorithm is explained to design adaptive inverse controller. Simulation results and their discussion for a dual input and dual output system is presented in Section 6 while using NN based adaptive inverse control scheme. Plant identification algorithm of RLS method and adaptive inverse control scheme is discussed in Section 8 . Also the obtained simulated results are compared for both plant parameter estimation and tracking trajectory performance evaluation in this Section. The Section 8 concluded the investigation. 


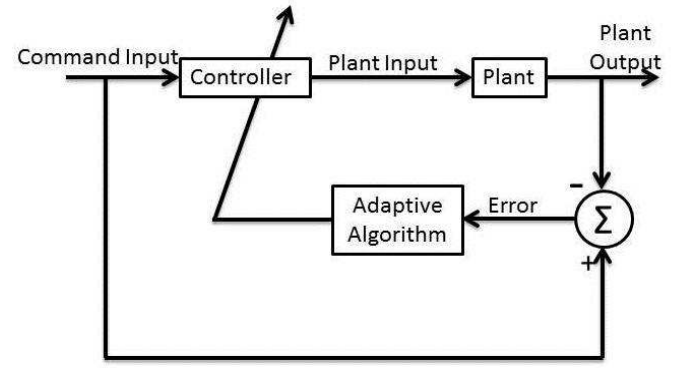

Fig. 1. Schematic diagram of basic adaptive inverse control

\section{Statement of Problem}

Considering a multi input and multi output (MIMO) discrete time linear system described by:

$$
y(z)=P(z) u(z)+V(z)
$$

where, multi outputs

$y(z)=\left[\begin{array}{lllllll}y_{1}(z) & y_{2}(z) & y_{3}(z) & - & - & - & y_{M}(z)\end{array}\right]^{T}$

multi inputs

$u(z)=\left[\begin{array}{lllllll}u_{1}(z) & u_{2}(z) & u_{3}(z) & - & - & - & u_{N}(z)\end{array}\right]^{T}$

disturbances

$V(z)=\left[\begin{array}{lllllll}V_{1}(z) & V_{2}(z) & V_{3}(z) & - & - & - & V_{M}(z)\end{array}\right]^{T}$

and the discrete transfer function

$$
P(z)=\left[\begin{array}{cccccc}
P_{11}(z) & P_{12}(z) & P_{13}(z) & - & - & P_{1 N}(z) \\
-- & -- & - & - & - & -- \\
-- & -- & -- & - & - & -- \\
-- & -- & -- & - & - & -- \\
P_{M 1}(z) & P_{M 2}(z) & P_{M 3}(z) & - & - & P_{M N}(z)
\end{array}\right]
$$

In the above equations, $u(z)$ is the inputs and $y(z)$ is the outputs of the measurable plant while $V(z)$ denotes for bounded disturbances. $P(z)$ is the discrete transfer function metrics. The aim of the neural network based inverse adaptive is to obtain a set of control inputs which are bounded. With the impact of these bounded control inputs, the outputs $y(z)$ should follow the reference inputs.

\section{ARCHITECTURE OF AdAPTIVE INVERSE CONTROLLER}

Adaptive inverse controller is not similar to the traditional closed loop controllers. The main concept of inverse adaptive control is to govern the system with a control command from the controller. The controller transfer function is the inverse of plant transfer function. The principal idea of inverse adaptive control is shown in Figure (1). Obtaining better tracking performance for the plant output is the main objective of this system. A true plant inverse need to be created by adapting the controller parameters because the plant is usually unknown. Comparing the plant output and command input, an error signal is produced to use for the adjusting process of the

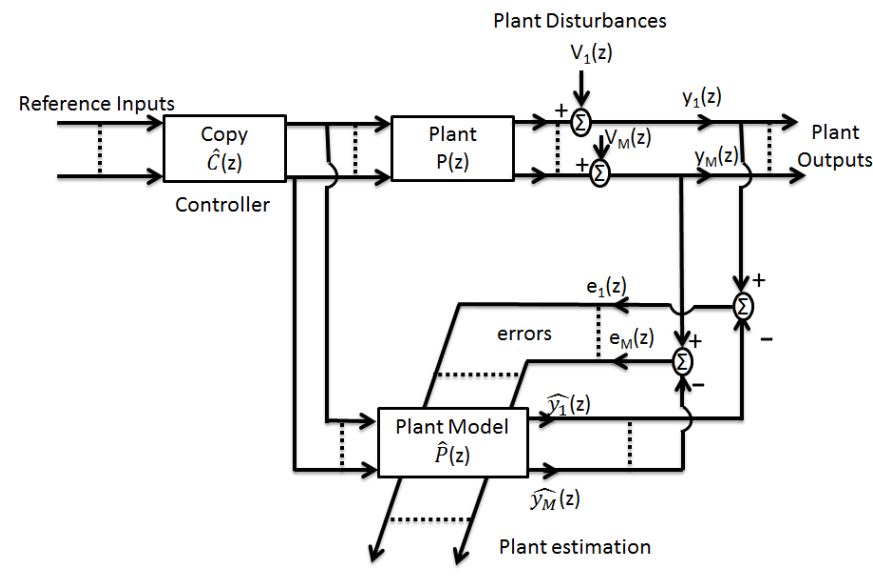

Fig. 2. Adaptive inverse- plant estimation for MIMO system

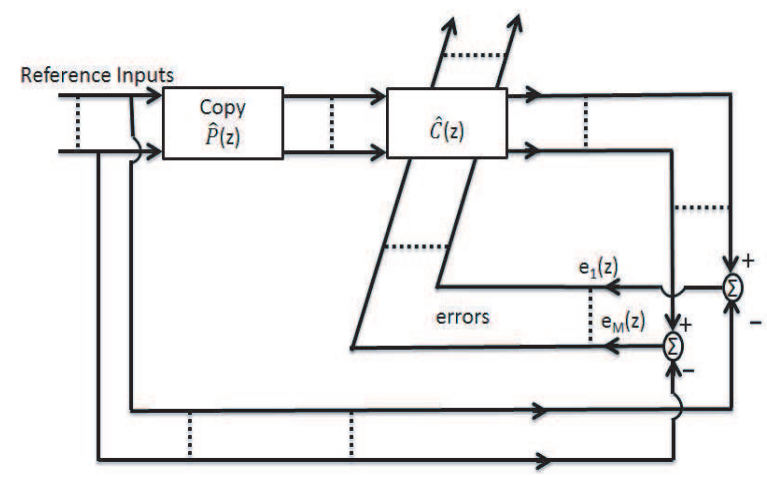

Fig. 3. Adaptive inverse- plant control for MIMO system

controller's parameters through an adaptive algorithm. Purpose of this algorithm is to minimize the error in terms of square mean. But this configuration has some demerits. Such as the adaptation process of the controller can not be done directly by the algorithm. Because the algorithm (for example, LMS) needs an error refereed to the plant input. Therefore a different configuration of adaptive inverse controller is proposed to overcome this problem and shown in Figure (2) and (3) for a MIMO system.

Rapid adaptation process and control action with plant disturbance represented in Figure (2) and (3). The plant identification and control mechanism are described as follows:

- Step 1: A MIMO plant model $\hat{P}(z)$ of the original plant $P(z)$ is identified on real time basis by using back propagating adaptive algorithm shown in Figure (2).

- Step 2: Updated parameters of controller $\hat{C}(z)$ is generated from a digital copy of $\hat{P}(z)$ either on-line or off-line and it is shown in Figure (3).

- $\quad$ Step 2: Finally the obtained updated $\hat{C}(z)$ can be used as a cascaded controller with the original plant $P(z)$ as presented in Figure (2). 


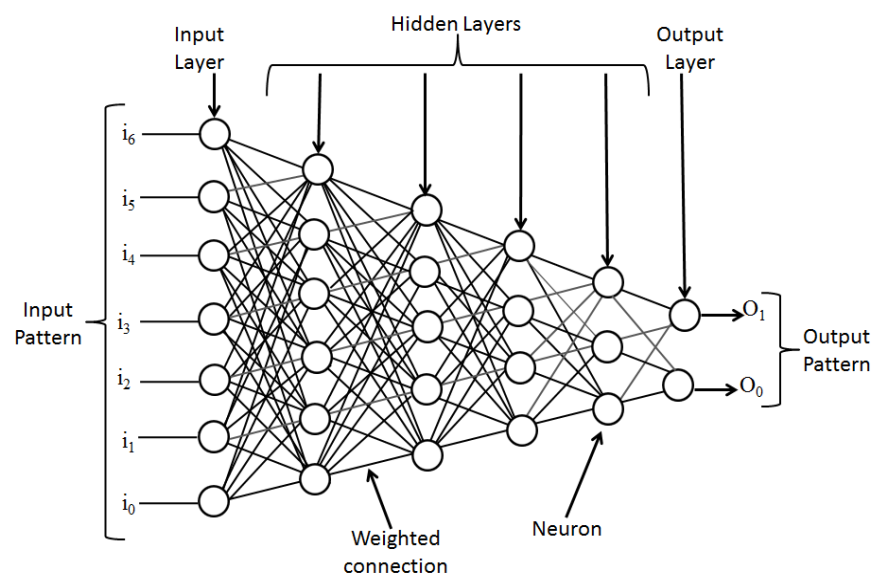

Fig. 4. Multi-layer neural network

\section{Neural Network with Multi Layer Perception}

A multi layer neural network contains multiple neurons, those are organized into different layers. The primary layers are placed at input side, the output layers are organized at the end and the middle area of the input and output is known as hidden layers [18]. It is already known that neural network is capable to execute in the presence of system nonlinearities because $\mathrm{NN}$ is a nonlinear filter. This property encourages to implement $\mathrm{NN}$ in the adaptive inverse problem. The neurons are connected towards forward direction without having any feed back connections between input and output. Therefore, in this work, the adaptive inverse control is implemented by using multi layer feed forward neural network (MLFFNN) [19] and shown in Figure (4). The active functions of successive layers can differ from each other. Connection link between input and neurons contain some weight. Neuron output is applicable to the nonlinear function.

\section{Adapting Controller Via Learning Algorithm}

The learning algorithm of this study is supported by back propagation technique for the NN based controller. The activation function induced by local field at the input is shown in Eq.(6). where, $y_{i}(n)$ is the output of $i$ th neuron for the $n$th iteration. The synaptic weight of the connecting link between output of $i$ th neuron and $j$ th neuron is denoted by $w_{j i}(n)$. The total number of inputs applied to the neuron $j$ is indicated by $m$.

$$
v_{j}(n)=\sum_{i=0}^{n} w_{j i}(n) y_{i}(n)
$$

The output at the $j$ th neuron is shown in Eq.(7) while the nonlinear function $\phi()$ is applied to the output of any neuron.

$$
y_{j}(n)=\phi_{j}\left(v_{j}(n)\right)
$$

The synaptic weights is updated according to the back propagation algorithm and it is expresses by the Eq.(8).

$$
w_{j i}(n+1)=w_{j i}(n)+\eta(n) \delta_{j}(n) y_{i}(n)
$$

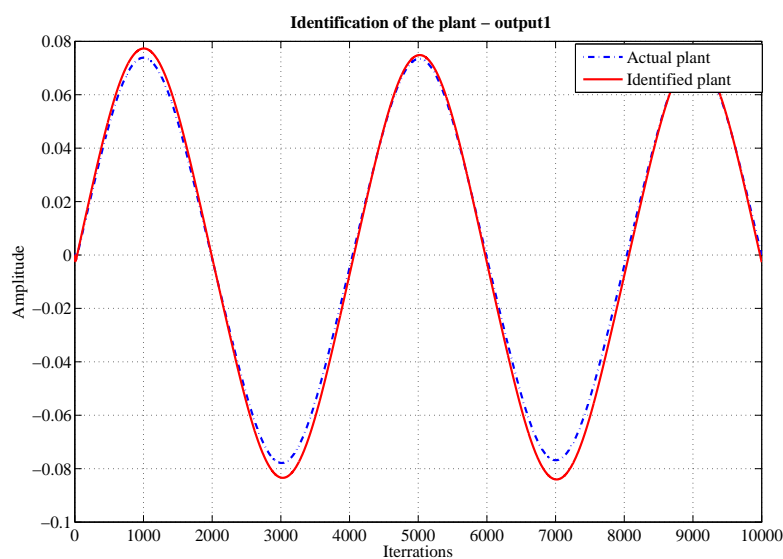

Fig. 5. MIMO plant identification without disturbance for output-1 (Sinusoidal input)

where $\delta_{j}$ is responsible for local gradient related with $j$ th neuron while the learning rate is denoted by $\eta(n)$. This learning rate is updated using following technique:

$$
\eta(n)=\frac{\psi(n)+\psi(n-1)+------\psi(n-m)}{m+1}
$$

here

$\psi=\alpha \eta(n-1)+\gamma\|e(n)\|^{2}$.

Mathematical expression of the local gradient $\delta_{j}$ is defined by Eq.(10) for $j$ output neuron.

$$
\delta_{j}(n)=e_{j}(n) \Phi_{j}^{\prime}\left(v_{j}(n)\right)
$$

where error $e_{j}$ is measured between the output and desired response $d_{j}(n)$. Again the local gradient can be calculated while the neuron comes from hidden layer and expressed by the Eq.(11).

$$
\delta_{j}(n)=\Phi_{j}^{\prime}\left(v_{j}(n)\right) \sum_{k} \delta_{k}(n) w_{k j}(n)
$$

\section{Simulation Results and Discussion}

Transfer function of a MIMO system is used for this investigation. To obtain direct and inverse model of MIMO system, we have used back propagating algorithm based on feed forward multi layer perception. The transfer function of double inputs and double outputs MIMO plant is shown in Eq. (12).

$$
P(z)=\left[\begin{array}{cc}
\frac{z^{-1}\left(0.1182-0.1531 z^{-1}\right)}{1-1.385 z^{-1}+0.4724 z^{-2}} & \frac{z^{-1}\left(0.1378-0.1378 z^{-1}\right)}{1-1.385 z^{-1}+0.4724 z^{-2}} \\
\frac{z^{-1}\left(-0.1174-0.09145 z^{-1}\right)}{1-1.385 z^{-1}+0.4724 z^{-2}} & \frac{z^{-1}\left(0.09867-0.1683 z^{-1}\right)}{1-1.385 z^{-1}+0.4724 z^{-2}}
\end{array}\right]
$$

\section{A. Plant identification}

Primarily the system is identified through adaptive inverse back propagation technique with random weight values while no disturbance is considered. Sinusoidal signal is given as the 


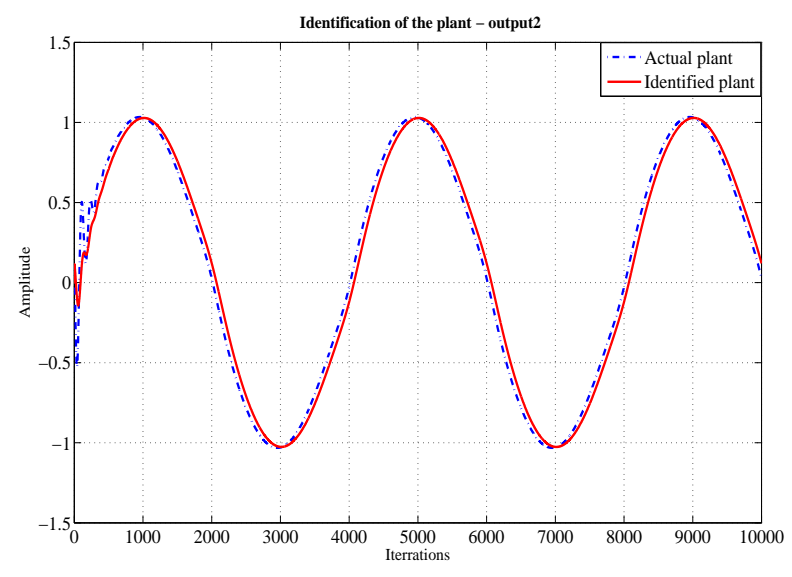

Fig. 6. MIMO plant identification without disturbance for output-2 (Sinusoidal input)

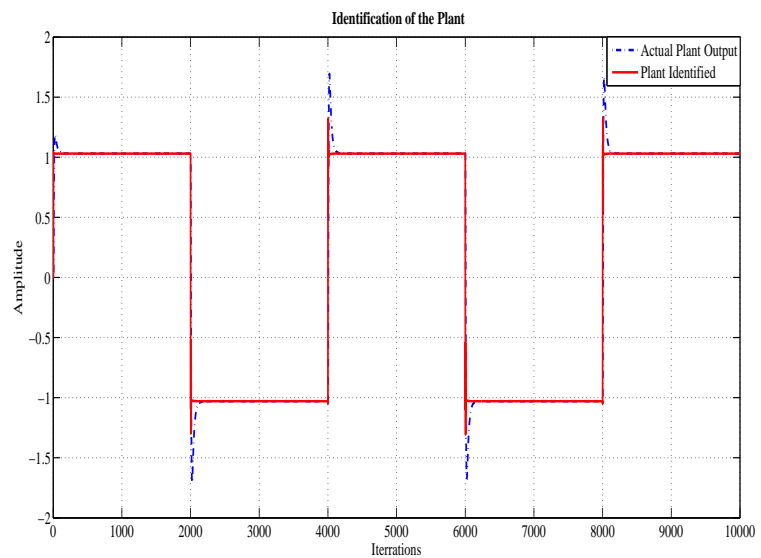

Fig. 7. MIMO plant identification for square input signal without disturbances (sample result)

reference input signal. Identified plant is shown in Figure (5) and (6) with respect to output-1 and output-2. It is observed that the plants are identified perfectly. To see the impact of changing the input signal the simulation was run again using square wave (reference input) as shown in Figure (7). Due to the changes of input signal the proposed technique found the plant identification nearly perfect.

The same simulation was repeated with random weight values in the presence of disturbance. With the same sinusoidal input signal, the identified plant is shown in Figure (8) and (9). Again the sample result of MIMO plant identification is shown in Figure (10) with disturbance condition while the input signal is changed from sinusoidal to square wave.

In both cases, an adaptive inverse with back propagation technique was found the satisfactorily identified system. The system was driven by uniform control signal. It is shown in the Figure (5) to (10) that the neural networks could be trained to identify the plant nearly perfect manner with and without disturbances. Usually with the disturbance, the plant dynamics should be disturbed. With the implementation of adaptive inversed based back propagation technique, the

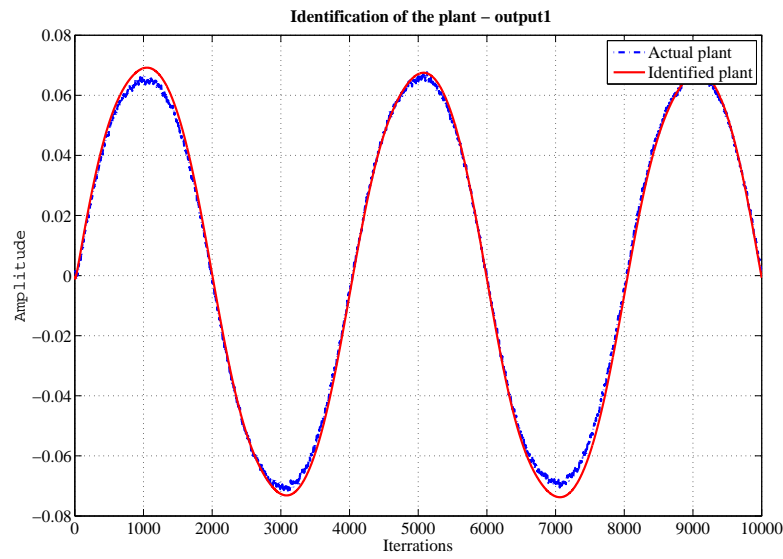

Fig. 8. MIMO plant identification with disturbance for output-1 (Sinusoidal input)

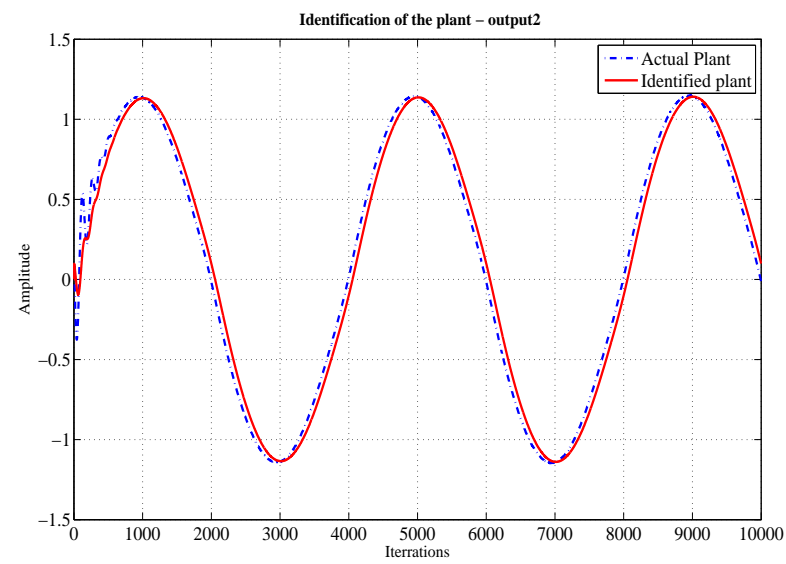

Fig. 9. MIMO plant identification with disturbance for output-2 (Sinusoidal input)

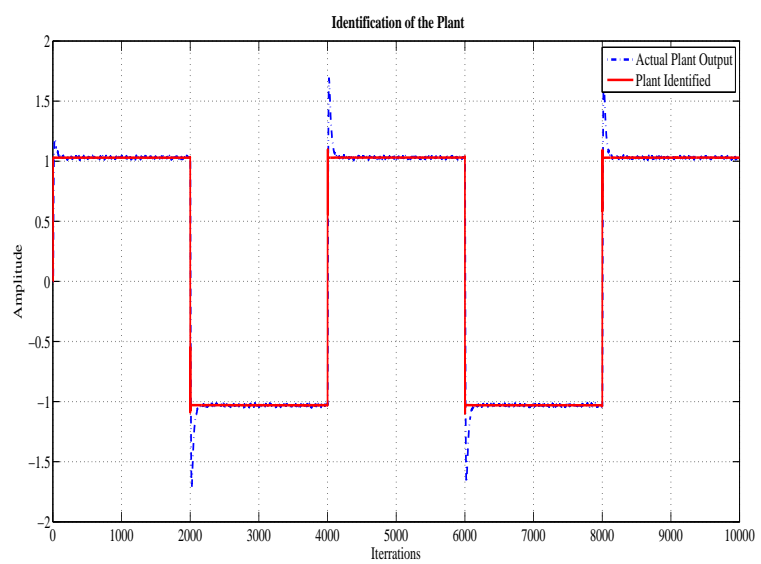

Fig. 10. MIMO plant identification for square input signal with disturbances (sample result) 


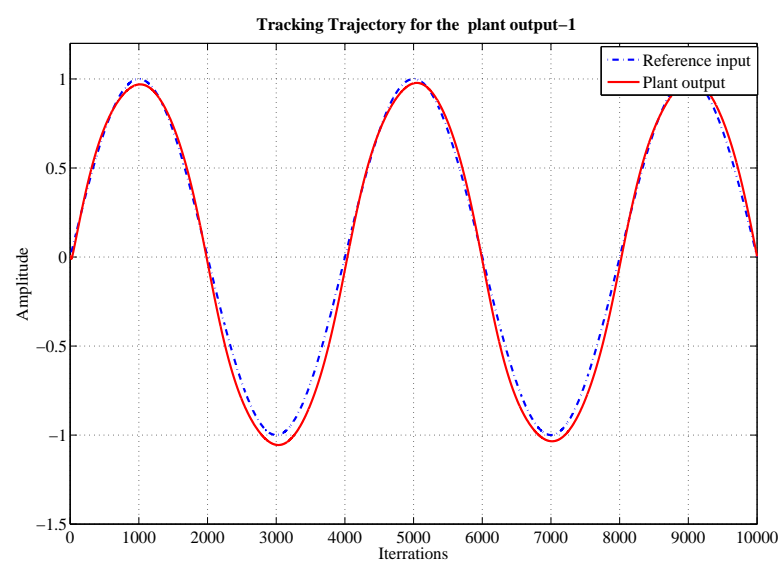

Fig. 11. Tracking trajectory for MIMO plant without disturbance for output-1

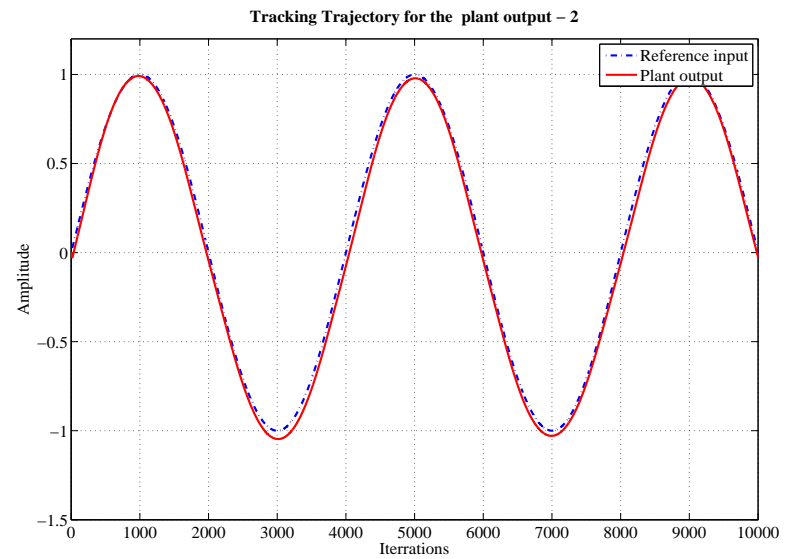

Fig. 12. Tracking trajectory for MIMO plant without disturbance for output-2

system identification processes matched the nominal dynamics of the plant. These proves the theoretical prediction.

\section{B. Plant control}

Once the plant identification is done then the control action is implemented using adaptive inverse technique to the MIMO system. Reference input is chosen as sinusoidal signal. Primarily the plant is experienced with no disturbances. The result is presented in Figure (11) and (11). The desired plant output ( blue dashed line) and the true system output (red solid line) are indicated in this result. Tracking of the sinusoidal input signal is nearly perfect while the plant does not experience any disturbances. A sinusoidal control signal is observed for this MIMO plant.

To observe the performance of disturbance cancellation, the disturbance signal is included in terms of noise in the algorithm. The filter is chosen for the purpose of disturbance cancellation. The effectiveness of the canceler was tested perfectly. The result is in shown Figure (13) and (14). The control signal of the MIMO plant is also sinusoidal while the disturbance is considered. To see the impact of changing the input signal to control the plant output, the simulation was run

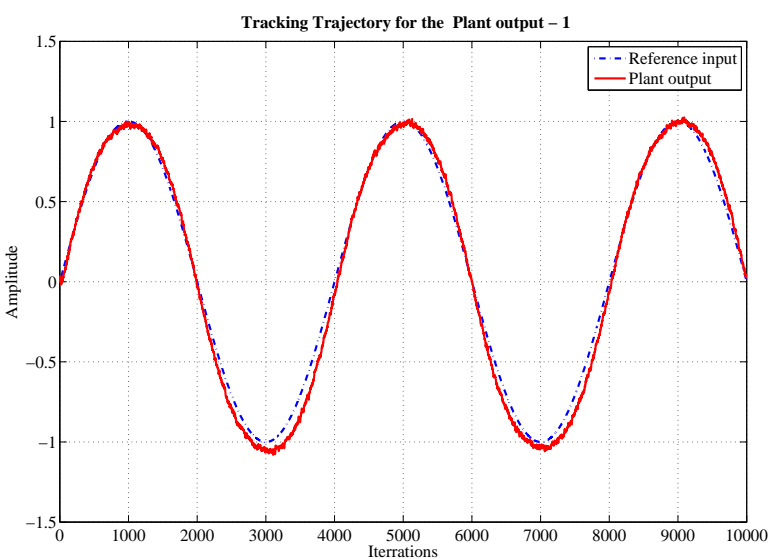

Fig. 13. Tracking trajectory for MIMO plant with disturbance for output-1

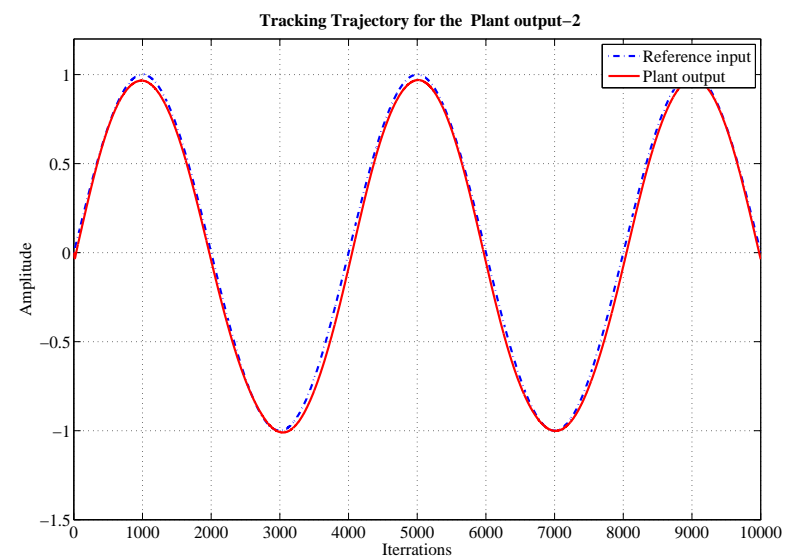

Fig. 14. Tracking trajectory for MIMO plant with disturbance for output-2

again using square wave (reference input) as shown in Figure (15).

\section{COMPARISON WITH RLS BASED ADAPTIVE INVERSE CONTROL Algorithm}

\section{A. Recursive Least Squares (RLS) Method}

The principle task of Recursive Least Squares (RLS) method is to calculate the state variables and observation vectors of the system. Then it compares between observation and the actual output of system. Finally it calculates the sum of squared errors. The parameter matrix is identified through a continuous modification process while the sum of squared error is achieved at its minimum range. Therefore, the identified parameters are kept closer to the actual parameters of the system [20]. Although RLS method is very fast process but it is highly complex in terms of computational cost.

\section{B. Summary of Identification Algorithm}

Multi order filter is considered to summarize RLS algorithm. In the Fig. 16, $r(m), y(m), d(m)$ and $z(m)$ are input, output, disturbance noise and measured output respectively. 


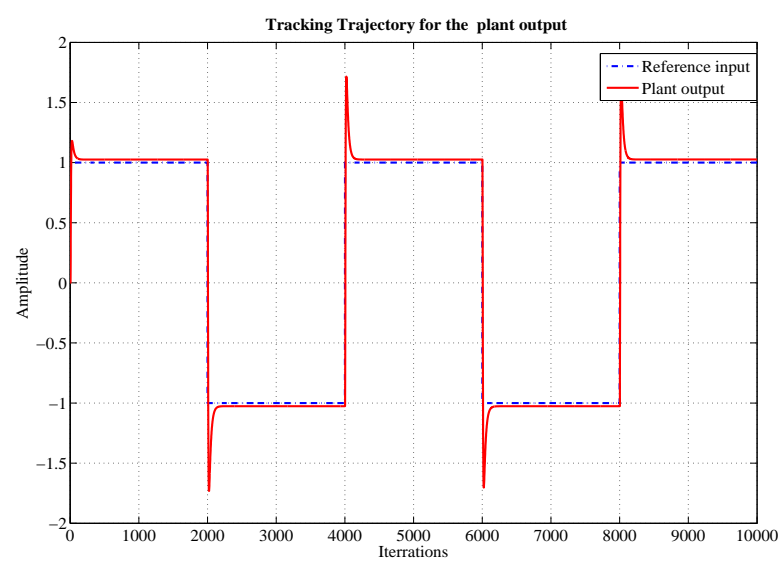

Fig. 15. Tracking trajectory of MIMO plant for square input signal (sample result)

$\alpha(m)$ is model parameter which is unknown. The model input is defined as

$$
\begin{gathered}
r(m)=\left[r_{1}(m), r_{2}(m), \ldots r_{n}(m)\right]^{T} \\
\alpha=[\alpha, \alpha, \ldots \alpha]^{T}
\end{gathered}
$$

Output parameters of the model is

$$
z(m)=r^{T}(m) \alpha+d(m)
$$

The function of least square criterion is deduced by

$$
C(\alpha)=\sum_{m=1}^{n}\left[z(m)-r^{T}(m) \alpha\right]^{2}
$$

$\alpha$ is estimated for the minimum value of $C(\alpha)$ and then $\hat{\alpha}$ is called the parameter values of least square estimation. Now the recursive least square (RLS) method is expressed through

$$
\left\{\begin{array}{l}
\hat{\alpha}(m)=\left[r(m)^{T} r(m)\right]^{-1} r(m)^{T} z(m) \\
q(m)^{-1}=r(m)^{T} r(m)
\end{array}\right.
$$

Where $q(m)$ is symmetric matrix positively decrease with the increase of $y$. The derived formulas for recursive methods are as follows:

$$
\hat{\alpha}(m)=\hat{\alpha}(m-1)+M(m)\left[r(m)-\theta^{T} \hat{\alpha}(m-1)\right]
$$

Here, $M(m)$ is gain matrix and defined as:

$$
\begin{gathered}
M(m)=\frac{q(m-1) \theta(m)}{1+\theta^{T}(m) q(m-1) \theta(m)} \\
q(m)=\left[I-M(m) \theta^{T}\right] q(m-1) \\
C(m)=C(m-1)+\frac{\left[z(m-1)-r^{T}(m-1) \alpha(m-1)\right]^{2}}{1+\theta^{T}(m-1) q(m-1) \theta(m-1)}
\end{gathered}
$$

Therefore, the residual is expressed as:

$$
\gamma(m)=\frac{\left[z(m-1)-r^{T}(m-1) \alpha(m-1)\right]^{2}}{1+\theta^{T}(m-1) q(m-1) \theta(m-1)}
$$

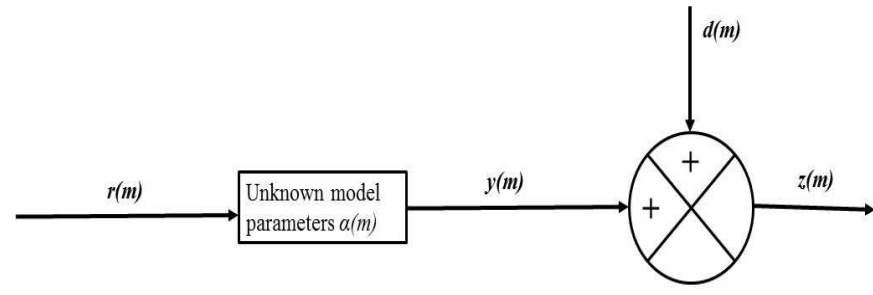

Fig. 16. Least Squares Method

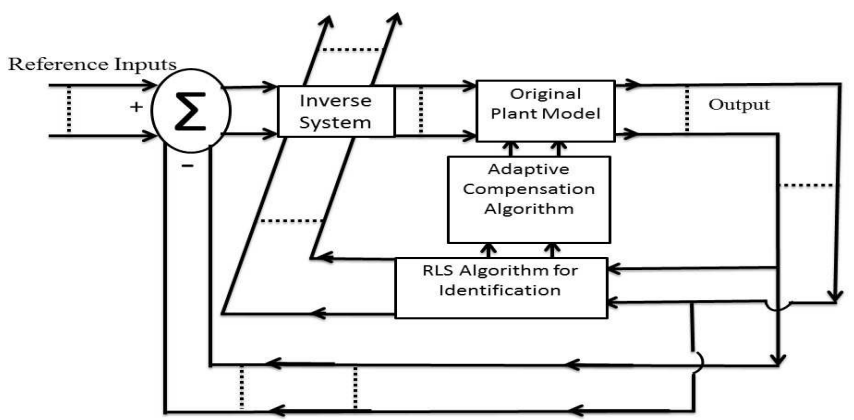

Fig. 17. RLS based adaptive inverse- plant control for MIMO system

Using suitable initial values for $q$ and $\hat{\alpha}$ recursive operation is performed so that the residual error $\gamma(m)$ is reduced enough. Hence, a minimum value is obtained for criterion function in order to complete the identification process.

\section{RLS Based Adaptive Inverse Control}

Figure 17 shows a schematic diagram of RLS based adaptive control for MIMO system. Structure contains several blocks like original model of the plant, inverse plant, adaptive algorithm, plant estimation algorithm, and feedback module. In this control architecture, plant is identified using RLS algorithm and expressed into S-function and converted into inverse system which combined with original plant connected in series. State feedback block forms a closed loop control architecture.

\section{Simulation results of RLS Based Adaptive Inverse Control - A comparison}

AThe same MIMO system defined in Eq. 12 is identified through RLS based adaptive inverse technique with random weight values. A square signal is given as the reference input signal. Identified plant is shown in Figure (18) and (19) with respect to output-1 and output-2. It is observed and compared with the result produced through back propagation based adaptive inverse control shown in Fig. 7 (without disturbance) and Fig. 10 (with disturbance). A better identification for plant parameters is obtained than RLS based estimation technique in terms of overshoot. Specifically, RLS based adaptive inverse algorithm of MIMO plant identification for square input signal with respect to output-2 contains very high overshoot. Therefore, the identified plant using neural network based controller is more perfect over RLS based estimation technique. 


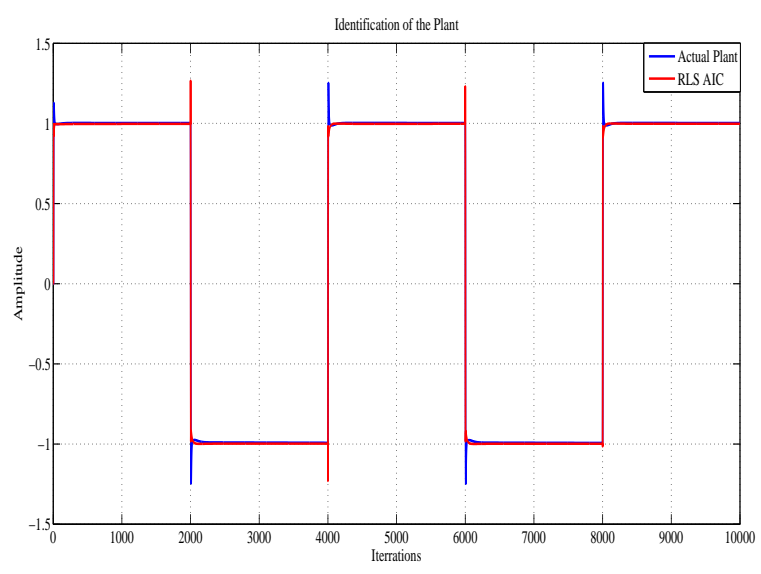

Fig. 18. MIMO plant identification for square input signal with respect to output-1 using RLS based adaptive inverse algorithm

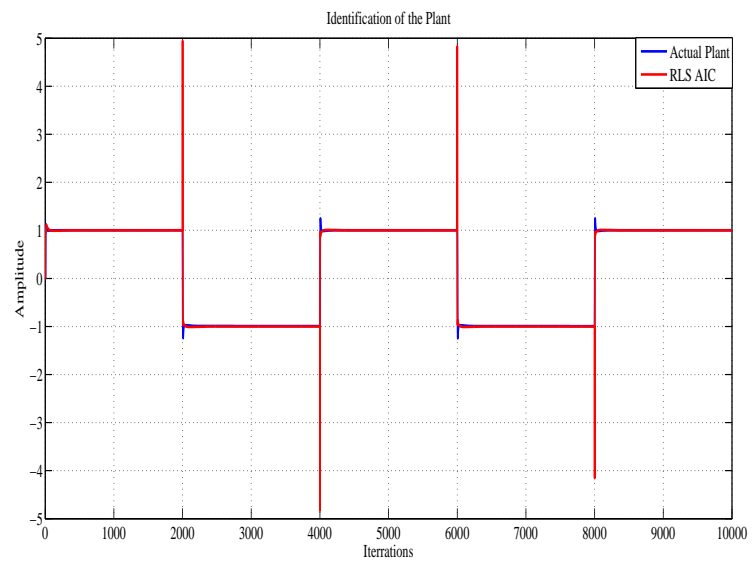

Fig. 19. MIMO plant identification for square input signal with respect to output-2 using RLS based adaptive inverse algorithm

Next step of plant parameter identification is to control the system. RLS based adaptive inverse technique is used to control the outputs of the same MIMO system. Reference input is chosen as square signal for this simulation. The plant controlled results are presented in Figure (20) and (21) with respect to output- 1 and output-2. The desired plant output ( blue dashed line) and the true system output (red solid line) are indicated in these results. It is observed that using RLS based adaptive inverse algorithm, tracking trajectory of MIMO plant for square input signal with respect to output- 2 contains very high overshoot. For the comparison with $\mathrm{NN}$ based adaptive inverse control technique, the plant is experienced with no disturbances which is shown in Fig. 15. It is found that the tracking trajectory of MIMO plant is more perfect while using back propagating algorithm based adaptive inverse control technique because its overshoot is with acceptable range. Due to the higher overshoot obtained in Fig. 21, it may cause instability of the system.

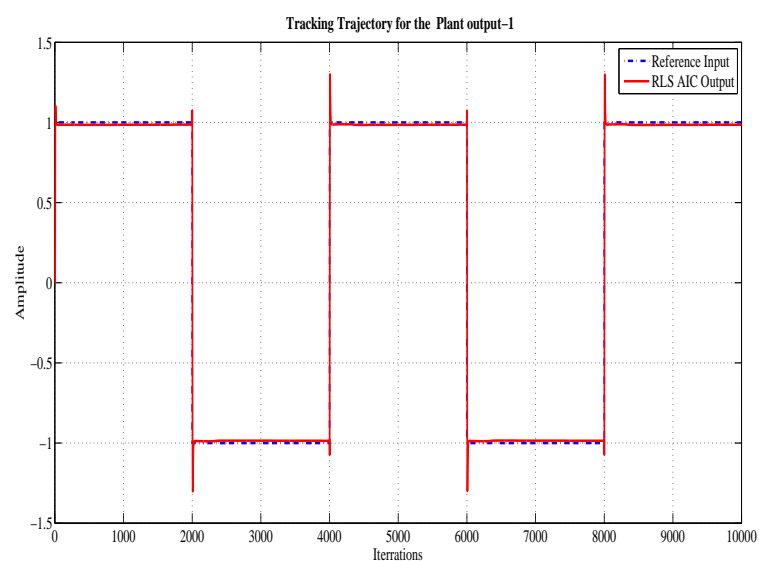

Fig. 20. Tracking trajectory of MIMO plant for square input signal with respect to output-1 using RLS based adaptive inverse algorithm

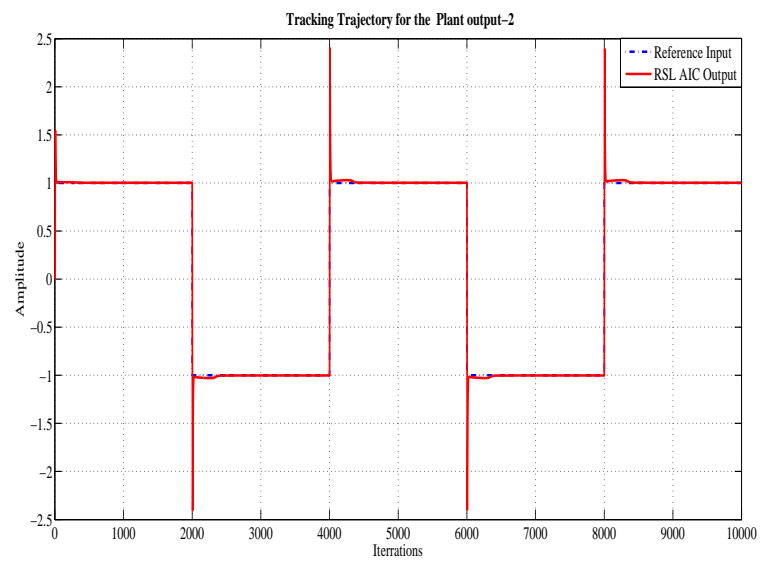

Fig. 21. Tracking trajectory of MIMO plant for square input signal with respect to output- 2 using RLS based adaptive inverse algorithm

\section{CONCLUSION}

In this paper, back propagation based adaptive inverse control technique is proposed to find the approximate inverse of the system. It has been shown that the proposed control method can perform well for MIMO system. It has also been shown nearly perfect performance while the disturbance is injected in term of noise. Therefore, the results verify the ability of neural network based adaptive inverse technique to control MIMO system. To prove the superiority of the proposed technique, the performance is compared with recursive least square (RLS) method for the same MIMO system. Plant identification algorithm of RLS method and adaptive inverse control scheme is discussed in this paper. Also the obtained simulated results are compared for both plant parameter estimation and tracking trajectory performance.

\section{ACKNOWLEDGMENT}

This article was funded by the Deanship of Scientific Research (DSR), King Abdulaziz University, Jeddah. Therefore, the authors acknowledge with thanks DSR financial support. 


\section{REFERENCES}

[1] S. Alkhalaf, Improvement of Control System Performance by Modification of Time Delay, (IJACSA) International Journal of Advanced Computer Science and Applications, 6(2), pp. 181-185, 2015.

[2] B. Audone, M. Audone and I. Marziali, On the use of the minimum phase algorithm in EMC data processing, International Symposium on Electromagnetic Compatibility (EMC EUROPE), pp. 1-6, 2012.

[3] J. Lu, M. Shafiq and T. Yahagi, A design method of model reference adaptive control for SISO non-minimum phase continuous-time systems using approximate inverse systems, Transaction IEEE of Japan, 117-C(3), pp. 315-321, 1997.

[4] J. Lu, M. Shafiq and T. Yahagi, A design method of model reference adaptive control for SISO non-minimum phase continuous-time systems based on pole-zero placement, IEICE Transaction Fundamentals, E80A(6), pp. 1109-1115, 1997.

[5] B. H'mida and S. Dhaou, Discrete-Time Approximation for Nonlinear Continuous Systems with Time Delays, (IJACSA) International Journal of Advanced Computer Science and Applications, 7(5), pp. 431-437, 2016.

[6] K. Astrom and M. B Witten, Adaptive control, Addison-Wesely, New York, 1995.

[7] J. Lu, M. Shafiq and T. Yahagi, A new method for self tuning control of non-minimum phase discrete-time systems in the presence of disturbances, Transaction IEEE of Japan, 117-C(2), pp. 110-116, 1997.

[8] A. Peiman, K. Abdollah and $\mathrm{H}$. Khayrollah, A novel adaptive fullydifferential GM-C filter, tuneable with a CMOS fuzzy logic controller for automatic channel equalization after digital transmissions, AEU International Journal of Electronics and Communications, 63(5), pp. 374386, 2017.

[9] A. R Maouche and H. Meddahi, A Fast Adaptive Artificial Neural Network Controller for Flexible Link Manipulators, (IJACSA) International Journal of Advanced Computer Science and Applications, 7(1), pp. 298308, 2016.

[10] T. Yahagi and J. Lu, On self-tuning control of non minimum phase discrete time systems using approximate inverse systems, Journal of Dynamic Systems, Measurement, and Control, Transaction AMSE, 115, pp. 12-18, 1993.

[11] K. Astrom and M. B Witten, self-tuning controller based on pole-zero placement, IEEE Proceedings. 120-D, pp. 120-130, 1980.

[12] B. Widrow and G. L Plett, Adaptive inverse control based on linear and nonlinear adaptive filtering, Proceedings of International Workshop on Neural Networks for Identification, Control, Robotics and signal/image processing, Venice, Italy, pp. 30-38, 1996.

[13] G. L Plett, Adaptive inverse control of plants with disturbance, $\mathrm{PhD}$ Thesis, Stanford University, Stanford, CA, 1998.

[14] B. S Kim and A. J Calise, Nonlinear flight control using neural networks, Journal of Guidance Control and Dynamics, 20(1), pp. 26-33, 1997.

[15] L. Yan and C. J Li, Robot learning control based of recurrent neural network inverse model, Journal of Robotic Systems, 14(3), pp. 199-212, 1997.

[16] K. S Narendra and K. Parthasarathy, Identification and control of dynamical system using neural networks, IEE transaction, Neural Network, 1(1), pp. 4-27, 1990.

[17] D. L Tien, H. J Kang, Y. S Suh and Y. S Ro, An online self-gain tuning method using neural networks for nonlinear PD computed torque controller of a 2-dof parallel manipulator, Neurocomputing, 116, pp. 53-61, 2013

[18] F. Laurene, "Fundamentals Of Neural Networks Architectures, Algorithms and Applications, Prentice Hall, ISBN:0133341860, 9780133341867, 1994.

[19] H. S Adheed and A. Sulaiman, Multi-Layer Feed Forward Neural Network Application In Adaptive Beamforming Of Smart Antenna System, International Conference on Multidisciplinary in IT and Communication Science and Applications (AIC-MITCSA), DOI: 10.1109/AICMITCSA.2016.7759925, pp. 1-6, 2016.

[20] Z. Jianhui and C. Siqin Adaptive Inverse System Control of Electromagnetic Linear Actuator, International Journal of Control and Automation, http://dx.doi.org/10.14257/ijca.2015.8.12.12, 8(12), pp. 131-144, 2015. 